\title{
Diferencias de género en percepciones sobre violencia sexual, igualdad y agresiones sexuales facilitadas por drogas en ocio nocturno
}

\section{Gender-based differences in perceptions about sexual violence, equality and drug-facilitated sexual assaults in nightlife contexts}

\author{
Pablo Prego-Meleiro*,**, Gemma Montalvo*,**, Carmen García-Ruiz****, \\ Fernando Ortega-Ojeda***, Isabel Ruiz-Pérez********, Luis Sordo**********. \\ * Departamento de Química Analítica, Química Física e Ingeniería Química. Universidad de Alcalá, Alcalá de Henares, España. \\ ** Instituto Universitario de Investigación en Ciencias Policiales (IUICP). Universidad de Alcalá, Alcalá de Henares, España. \\ *** Escuela Andaluza de Salud Pública (EASP), Granada, España. \\ **** CIBER en Epidemiología y Salud Pública (CIBERESP), Madrid, España. \\ ***** Departamento de Salud Pública y Salud Materno-infantil. Universidad Complutense de Madrid, España.
}

\section{Resumen}

La violencia sexual, incluyendo las agresiones sexuales facilitadas por drogas, es un serio problema cada vez más común en los contextos de ocio nocturno. Este trabajo estudia las actitudes y percepciones de la juventud española en torno a la violencia sexual en dicho ámbito. Los participantes se reclutaron mediante muestreo en bola de nieve. Se realizó un análisis bivariado para identificar posibles factores sociodemográficos y de ocio nocturno relacionados con género. La significación estadística de las diferencias entre estas variables se evaluó mediante las pruebas de chicuadrado y exacta de Fisher. Las mujeres percibieron un menor nivel de seguridad personal, así como la existencia de percepciones sociales que penalizan en mayor medida el consumo de drogas femenino que el masculino, y que culpabilizan a las mujeres por la violencia que sufren. Además, ellos mostraron mayor disposición a mantener relaciones sexuales con personas incapaces de otorgar su consentimiento debido a los efectos de las drogas. Los hombres creen en mayor medida que las agresiones sexuales ocurren debido al uso de alcohol $\mathrm{u}$ otras drogas por parte de las víctimas. En el contexto de ocio nocturno, las mujeres son propensas a percibir la existencia de una falta de apoyo social hacia ellas, así como un sentimiento de impunidad social hacia los agresores. Además, existen numerosas concepciones erróneas en torno a las agresiones sexuales facilitadas por drogas. La mayoría cree que las agresiones ocurren tras la administración encubierta de sustancias a la víctima por parte de un agresor desconocido. Además, se subestimó la participación del alcohol. Nuestros hallazgos son útiles para diseñar esfuerzos preventivos bien dirigidos, desmitificar el fenómeno de las agresiones sexuales facilitadas por drogas y mejorar el apoyo social a las víctimas.

Palabras clave: Agresión sexual facilitada por drogas; violencia sexual; prevención juvenil; mitos de la violación; violencia cultural.

\begin{abstract}
Sexual violence, including drug-facilitated sexual assaults, is a serious issue that is becoming increasingly common in leisure nightlife contexts. This study provides information about the attitudes and perceptions of Spanish youths towards sexual violence within that setting. The participants were recruited by a snowball sampling scheme. A bivariate analysis was performed to identify possible sociodemographic and nightlife recreational habit factors related to gender. The statistical significance of the differences between the studied variables was assessed using the chi-squared and Fisher's exact tests. Women perceived a low level of personal security, as well as the existence of social perceptions penalizing female more than male drug use, and blaming women for the sexual violence they suffer. Women also recognised less explicit violent behaviours as violence significantly more than men did. Men were more willing to have sexual intercourse with someone unable to express consent because of drugs. In addition, they believed more strongly that sexual assaults take place because of the victim's use of alcohol or other drugs. In a leisure nightlife context, women are prone to perceive a lack of social support for themselves and the feeling of impunity for the perpetrators. Furthermore, numerous misconceptions surround drugfacilitated sexual assaults, with the majority of respondents believing that assaults happen after the surreptitious administration of substances to the victim by an unknown assailant. Moreover, the involvement of alcohol was underestimated. Our findings are useful for designing prevention efforts, demystifying the drug-facilitated sexual assaults and enhancing social support for victims.
\end{abstract}

Keywords: Drug-facilitated sexual assault; sexual violence; youth prevention; rape myths; cultural violence.

Recibido: Mayo 2020; Aceptado: Diciembre 2020.

Enviar correspondencia a:

Pablo Prego Meleiro. Universidad de Alcalá, Alcalá de Henares, España.

Email: pregomeleiro.research.uah@gmail.com 


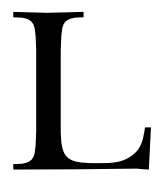
a violencia sexual es una forma de violencia interpersonal, típicamente dirigida hacia las mujeres, que incluye cualquier acto sexual, comentarios o insinuaciones sexuales no deseadas. Como tal, la violencia sexual representa uno de los problemas más graves de salud pública y derechos humanos en todo el mundo (Organización Mundial de la Salud, 2013, 2014). Alrededor del $11 \%$ de las mujeres europeas entre 18 y 74 años han sufrido violencia sexual en algún momento de su vida, con el $5 \%$ habiendo sido sometidas a relaciones sexuales no consentidas mientras las sujetaban o les hacían daño de alguna manera (European Union Agency for Fundamental Rights, 2014). Por grupo de edad, son particularmente afectadas las mujeres jóvenes y en edad universitaria (Bird, Gilmore, George y Lewis, 2015; Carey, Durney, Shepardson y Carey, 2015; Krebs, Lindquist, Warner, Fisher y Martin, 2007). En España, de acuerdo a una investigación reciente sobre violencia contra las mujeres en una población universitaria (Valls, Puigvert, Melgar y Garcia-Yeste, 2016), sólo el 56\% de las mujeres y el $42 \%$ de los hombres consideraron todas las situaciones de violencia presentadas como violencia. Asimismo, la prevalencia más alta de la violencia sexual en este país durante el último año afectó a mujeres entre 16-29 años de edad (Government Delegation against Gender Violence, 2015). Sin embargo, la mayoría de los incidentes de violencia sexual no son reportados, por lo que los estudios epidemiológicos son de particular importancia para hacerse una idea de la magnitud de este problema en diferentes poblaciones (Hellmann, Kinninger y Kliem, 2018).

Ciertas percepciones, actitudes y normas sociales contribuyen a la configuración de la violencia sexual. Esto implica, hasta cierto punto, que la cultura define los límites entre conductas aceptables y abusivas (Government Delegation for National Plan on Drugs, 2018; Organización Mundial de la Salud, 2002). En esta línea, la violencia cultural se refiere a los aspectos culturales que justifican la violencia directa o estructural y que reprimen las respuestas de las víctimas (Galtung, 1990). Una sociedad que no percibe los diversos tipo de violencia sexual no solo no los va a reprimir ni perseguir, sino que, y lo que es más importante, será incapaz de implementar políticas de prevención, en particular entre los jóvenes (Sasson y Paul, 2014). Existe una brecha entre la población en general y las personas involucradas activamente en el campo de la violencia sexual, en lo que se refiere a la percepción de las definiciones y causas de la violencia sexual, y también respecto de las ideas sobre víctimas y agresores (O’Neil y Morgan, 2010). De hecho, los estudios sobre violencia sexual muestran que la población en general parece identificar actos específicos, como la violación, como violencia sexual, pero no las formas más sutiles, como el lenguaje sexualmente degradante (sobre las mujeres) o el acoso (McMahon y Farmer, 2011). Estas diferencias pueden deberse, en parte, a diferentes puntos de vista sobre las causas de la violencia sexual (McMahon, 2010). En España, la única encuesta que ha estudiado la percepción social de la violencia sexual observó la invisibilidad de las conductas violentas distintas de las relaciones sexuales no deseadas. Este estudio señaló que la mayoría de los jóvenes se encuentra a medio camino entre el rechazo y la tolerancia del sexismo, y también encontró diferencias significativas entre sexos en la percepción de la violencia sexual (Government Delegation against Gender Violence, 2018). Sin embargo, este estudio prestó poca atención a la relación entre la violencia sexual y el consumo de drogas. Existe en la actualidad una marcada convergencia en las relaciones de género en contextos de ocio nocturno que puede aumentar las consecuencias negativas para las mujeres (Calafat et al., 2003; Calafat, Juan, Becona, Mantecon y Ramon, 2009; Gilbert y Pearson, 2003; Hughes et al., 2011). Así, las mujeres jóvenes han comenzado a incidir en espacios tradicionalmente masculinos ante la ausencia de una verdadera igualdad de género (Prego-Meleiro, Montalvo, Quintela-Jorge y García-Ruiz, 2020a). Como ejemplo, la prevalencia de binge drinking ha aumentado de manera constante en las mujeres desde la segunda mitad de la década de 1990, acercándose a los niveles típicamente hallados para varones (Government Delegation for National Plan on Drugs, 2018).

La violencia sexual también ocurre cuando alguien es incapaz de dar su consentimiento o rechazar una propuesta sexual debido a los efectos de sustancias psicoactivas (Organización Mundial de la Salud, 2002). Esto ocurre en las agresiones sexuales facilitadas por las drogas (DFSA) (Advisory Council of the Misuse of Drugs, 2007). Se trata de una forma interseccional de violencia sexual (Prego-Meleiro, Montalvo, Quintela-Jorge y García-Ruiz, 2020b) particularmente común en contextos recreativos (Folgar, Rivera, Sierra y Vallejo-Medina, 2015; Lawyer, Resnick, Bakanic, Burkett y Kilpatrick, 2010; Resnick, Walsh, Schumacher, Kilpatrick y Acierno, 2012), ya que en estas situaciones convergen víctimas potenciales, y agresores motivados en la ausencia de tutores capaces (Mustaine y Tewksbury, 2002). En España, las fiestas y los festivales son considerados como los principales contextos en los que se ocurren agresiones sexuales contra las mujeres (Government Delegation against Gender Violence, 2018). Sin embargo, poco se sabe acerca de la percepción habitual de la población sobre DFSA o de cualquier diferencia de sexo la percepción de este problema. La violencia cultural implica reacciones sociales negativas, incluyendo la culpabilización de la víctima, lo que dificulta el autorreconocimiento de experiencias sexuales no deseadas (Bondurant, 2001; Fisher, Daigle, Cullen y Turner, 2003), desencadena problemas de salud psicológica en las víctimas (Ullman y Filipas, 2001; Ullman y Najdowski, 2010) y aumenta el riesgo de revictimización (Lorenz y Ullman, 2016). En ese marco, este estudio sugiere las siguientes hipótesis: (i) las percepciones 
de igualdad, seguridad y riesgo de sufrir violencia sexual en contextos de ocio nocturno varían significativamente en función del género; (ii) existe una brecha social entre los géneros en la identificación de algunos actos específicos como violencia sexual; (iii) la disposición a mantener relaciones sexuales con alguien que no puede expresar su consentimiento debido los efectos provocados por las drogas es mayor entre los hombres que entre las mujeres; (iii) existen varios mitos o conceptos erróneos generalizados sobre el fenómeno DFSA que afectan significativamente a la concepción social de esta forma de violencia sexual; y (v) las mujeres experimentan una falta de apoyo social en contextos de ocio nocturno debido a las diferentes percepciones sociales sobre el uso de drogas en función del género de la persona consumidora.

En el contexto de la igualdad, la modificación de supuestos incorrectos y expectativas culturales sobre la violencia sexual es un paso clave para reducir este problema. Para ello, la universidad es un contexto de especial interés porque muestra el entorno cultural de las generaciones más jóvenes. Por lo tanto, este trabajo tuvo como objetivo determinar las percepciones de género sobre diferentes aspectos de la violencia sexual en el contexto del ocio nocturno, especialmente la normalización y justificación de la violencia, prestando especial atención al papel que juega el consumo de drogas. Este objetivo se alinea con la necesidad de aumentar la conciencia social sobre el fenómeno DFSA como una forma de violencia sexual que afecta severamente a las mujeres en el contexto de la vida nocturna de ocio.

\section{Materiales y métodos}

\section{Sujetos}

Los participantes fueron reclutados mediante un esquema de muestreo de bola de nieve utilizando un cuestionario en línea cerrado de dos fases. Primero, completaron el cuestionario 229 estudiantes de la Universidad de Alcalá (España) que participaron en el proyecto educativo UAH/ EV951. Se les dieron instrucciones a los estudiantes para enviar el mismo cuestionario a un mínimo de diez contactos pertenecientes a su círculo inmediato, utilizando los servicios de mensajería instantánea disponibles a través de las redes sociales. Los participantes de esta fase fueron instruidos adecuadamente sobre los criterios de inclusión del estudio; tener entre 16 y 35 años y residir en España en el momento del estudio. Su participación fue completamente anónima a través de dispositivos electrónicos, como teléfonos móviles u ordenadores. Este proceso se llevó a cabo entre octubre y diciembre 2017. Después del muestreo inicial, se excluyó a cualquier participante que no cumplía los requisitos. El tamaño muestral final fue de 2.355 jóvenes.

Durante el desarrollo del estudio, antes de acceder al cuestionario en línea, todos los participantes leyeron un documento previo donde se les informó en detalle acerca de la razón y de los propósitos de la investigación, así como sobre los criterios de inclusión del estudio. Asimismo, dicho documento previo presentó a los participantes los temas abordados por el cuestionario antes de que pudieran acceder al mismo, así como una estimación del tiempo necesario para completarlo (15 minutos). Esta pestaña anterior solicitó a los participantes un consentimiento para su participación voluntaria y anónima antes de acceder al cuestionario, lo que constituía el consentimiento positivo para su participación. El acceso a la encuesta se brindó únicamente a través de un enlace disponible tras la presentación de la información indicada. Se prestó especial atención al diseño de las preguntas para evitar obtener datos identificativos de los participantes. Por ejemplo, se les pidió informar de su edad en años en lugar de su fecha de nacimiento, y no hubo pregunta alguna sobre su lugar de residencia o procedencia geográfica. Además, no se recogieron las direcciones IP de Internet ni las cookies. La comisión evaluadora de la «Convocatoria de Proyectos de Promoción de la Innovación Docente en el Proceso de Enseñanza-Aprendizaje» de la Universidad de Alcalá (curso 2017-18) aprobó la ejecución del proyecto en el que se enmarca este estudio (número de referencia UAH/EV951). La aprobación del proyecto dentro de este marco institucional requiere el cumplimiento obligatorio de requisitos éticos específicos.

\section{Recolección de datos y medidas}

Las variables estudiadas se recopilaron en cuatro grupos: sociodemográficas; hábitos de ocio nocturno, incluido el uso de drogas; percepciones sobre la violencia sexual e igualdad en el contexto de ocio nocturno; y percepciones sobre DFSA en el contexto de ocio nocturno.

Sociodemográficas. La edad se midió como una variable estratificada siguiendo los rangos más frecuentes utilizados en estudios similares: menores de 18 años (edad preuniversitaria), 18-24 años (edad universitaria) y > 24 años. Otras variables fueron: nivel de estudios y nivel de estudios de los padres (universidad/ciclo superior; bachillerato/grado medio o básico/sin estudios) e ingresos familiares autopercibidos. Las preguntas sociodemográficas fueron limitadas para evitar recolectar información que pudiera identificar a los estudiantes desde la primera fase; es decir, se garantizó el anonimato.

Hábitos de ocio nocturno. El cuestionario incluyó seis ítems, estructurados de la siguiente manera: frecuencia de salidas durante el último año; frecuencia de consumo de alcohol, cannabis, cocaína, anfetaminas o benzodiazepinas durante el tiempo de ocio, y nivel de importancia respecto al consumo de alcohol y otras drogas en el tiempo de ocio. En cuanto a la frecuencia de salidas, las opciones de respuesta incluyeron cinco categorías, desde «más de una vez a la semana» a «nunca». Se utilizaron otras cinco categorías 
de respuesta para preguntas sobre la frecuencia de uso de sustancias psicoactivas, agrupadas como «siempre o con frecuencia», «alguna vez o pocas veces» $\mathrm{y}$ «nunca». A su vez, la respuesta opcional de las preguntas centradas en el nivel de importancia del uso de sustancias incluyó otras cinco categorías, desde «indispensable» a «indiferente».

Percepciones sobre violencia sexual e igualdad en el contexto de ocio nocturno. Para asegurar la comparabilidad de nuestros hallazgos, las preguntas fueron adaptadas a partir de estudios previos. Aquellas relativas a igualdad, seguridad e impunidad se tomaron de estudios basados en encuestas y realizados en estudios con encuestas en España (Government Delegation against Gender Violence, 2015; Observatorio Noctámbul@S, 2017, 2018) y Europa (European Union Agency for Fundamental Rights, 2014). Las preguntas se centraron en las percepciones sobre la igualdad entre mujeres y hombres en la vida nocturna, incluido el nivel de seguridad, la preocupación por el riesgo de sufrir violencia sexual y las consecuencias legales para los agresores. Se incluyeron cinco ítems que requirieron una respuesta dicotómica ( sí o no). Como ejemplo, algunos ítems son: «¿Te preocupa el riesgo de violencia sexual cuando sales?», «¿Crees que los espacios de ocio nocturno son igualitarios para mujeres y hombres?». Las preguntas sobre la consideración de diversas conductas como formas de violencia sexual incluyeron violación, tocamientos no consentidos, acorralamiento, comentarios sexuales invasivos e insistencia frente a negativas. Estos ítems fueron seleccionados a partir de la Sexual Experiences Survey (SES) o Encuesta de Experiencias Sexuales (Koss et al., 2006), estudios españoles anteriores (Observatorio Noctámbul@S, 2017, 2018) así como las definiciones de la Organización Mundial de la Salud. La Encuesta de Experiencias Sexuales de victimización de formato largo (SES-LFV) es una versión de la SES que valora la victimización mediante encuentros sexuales no deseados, incluida la violación. Se pidió a los encuestados que marcasen las experiencias que consideran ser formas de violencia sexual, entre las siguientes: «violación», «tocamientos no consentidos», «acorralamiento», «comentarios sexuales invasivos» $\mathrm{e}$ «insistencia frente a negativas». Otras preguntas sobre normalización de la violencia sexual y la justificación de las relaciones sexuales forzadas también se basaron en los enfoques utilizados en las encuestas oficiales (European Union Agency for Fundamental Rights, 2014; Government Delegation against Gender Violence, 2015). Se incluyeron siete ítems con respuestas dicotómicas (si o no) respecto de las preguntas sobre normalización y justificación de la violencia sexual. Para el diseño de estos ítems, se utilizó la escala de Aceptación de Mitos Modernos sobre la Agresión Sexual (AMMSA) (Megías, Romero-Sánchez, Durán, Moya y Bohner, 2011); concretamente, los relacionados con la interseccionalidad entre violencia sexual y consumo de drogas. De este modo, el estudio sobre la normalización de la violencia incluyó cuatro ítems. Dos de esos ítems son: «¿Piensas que las mujeres que se divierten en ambientes de ocio nocturno deben asumir los riesgos de ser agredidas sexualmente?», «iPiensas que las agresiones sexuales ocurren debido al mayor uso de drogas por parte de las mujeres?». Otros tres ítems abordaron la justificación de las relaciones sexuales forzadas, por ejemplo: «¿Piensas que las relaciones sexuales forzadas están justificadas si una mujer está bajo los efectos de las drogas?».

Percepciones sobre DFSA. Los autores desarrollaron varias preguntas sobre DFSA debido a la falta de cuestionarios sobre este fenómeno en el momento del estudio. Revisiones anteriores del tema permitieron identificar ítems relevantes (Anderson, Flynn y Pilgrim, 2017; Lorenz y Ullman, 2016). Estas preguntas incluyeron ocho ítems que abarcan la disposición a realizar conductas DFSA; las prácticas de riesgo para la victimización por DFSA (compartir la propia bebida y aceptar bebidas con contenido desconocido); el tipo de consumo y el tipo de drogas, y la relación entre víctima y agresor en la mayoría de los casos DFSA. Además, se incluyeron otros cuatro ítems relacionados con la percepción social del consumo de drogas en función del género. En concreto, la existencia de una consideración igualitaria en cuanto al consumo de drogas masculino y femenino, la mayor normalización de las imágenes de hombres ebrios y la tolerancia social hacia hombres y mujeres cuando se encuentran bajo los efectos de las drogas; por último, el apoyo social en situaciones de riesgo, especialmente la penalización social hacia los hombres por intentar aprovecharse de una mujer que está experimentando dificultades motoras debido a los efectos provocados por las sustancias psicoactivas.

\section{Análisis de datos}

Todas las variables se describieron para toda la muestra y se estratificaron por género. Se realizó un análisis bivariable para identificar posibles factores sociodemográficos y hábitos de ocio nocturno relacionados con el género. La significación estadística de las diferencias entre estas variables se evaluó mediante las pruebas de chi cuadrado y exacta de Fisher. El nivel de significancia para las pruebas bilaterales se fijó en $p<, 05$.

Las diferencias basadas en género en las percepciones sobre violencia sexual, igualdad y DFSA en contexto de ocio nocturno se calcularon utilizando la razón de momios bruta (OR) y ajustada (ORa). La ORa fue determinada usando modelos de regresión logística. Estos modelos se ajustaron para evaluar que las diferencias en las percepciones basadas en género no se viesen afectadas por otros factores sociodemográficos o hábitos de ocio nocturno. Los modelos incluyeron todas las variables con $p<, 10$ en el análisis bivariable. También se evaluaron las posibles interacciones entre los factores relacionados. Se obtuvieron las OR, ORa y sus correspondientes intervalos de confianza de 95\% (IC del 95\%) como medidas de la fuerza de la relación. 


\section{Resultados}

De los 2.355 encuestados, el $66,0 \%$ eran mujeres, el $74,0 \%$ tenían una edad comprendida entre 18 y 24 años (edad media 20,6 años, $S D=4,2$ ), el 73,4\% tenían un nivel de estudios superior a los estudios básicos, el 40,3\% procedía de familias en las que al menos uno de los padres tenía estudios universitarios o superiores y el $66,7 \%$ procedía de familias con ingresos de nivel medio, bajo o muy bajo. Respecto de los hábitos de ocio nocturno durante los últimos 12 meses, el 64,3\% había salido al menos una vez al mes, consumiendo alcohol o cannabis siempre o frecuentemen- te el $72,8 \%$ y el $7,5 \%$, respectivamente. El $33,3 \%$, aproximadamente, pensaba que el consumo de alcohol y/u otras drogas era muy importante durante su tiempo de ocio. Las mujeres eran más jóvenes $(p<, 001)$, sus madres tenían un mayor nivel educativo $(p=, 016)$ y consumían menos cannabis y cocaína durante su tiempo de ocio $(p<, 001)$. La Tabla 1 muestra las características sociodemográficas.

La Tabla 2 muestra las percepciones de igualdad, seguridad e impunidad en los contextos de ocio nocturno, que fueron claramente diferentes para hombres y mujeres. De todos los encuestados, el $86,0 \%$ percibió los espacios de

Tabla 1. Resultados demográficos.

\begin{tabular}{|c|c|c|c|c|}
\hline & Total & Mujeres & Hombres & \\
\hline Edad $^{\star \star \star}$ & N (\%) & N (\%) & N (\%) & $p$ \\
\hline $16-17$ & $353(15,0)$ & $272(17,5)$ & $81(10,1)$ & \\
\hline $18-24$ & $1742(74,0)$ & $1129(72,6)$ & $613(76,6)$ & $<, 001$ \\
\hline$>24$ & $260(11,0)$ & $154(9,9)$ & $106(13,3)$ & \\
\hline \multicolumn{5}{|l|}{ Nivel de estudios } \\
\hline Universitarios y/o superiores & $854(36,5)$ & $557(35,8)$ & $297(37,5)$ & \multirow{3}{*}{,131 } \\
\hline Secundarios/secundarios superiores & $863(36,9)$ & $592(38,3)$ & $21(34,2)$ & \\
\hline Básicos o sin estudios & $623(26,6)$ & $398(25,7)$ & $225(28,4)$ & \\
\hline \multicolumn{5}{|l|}{ Nivel de estudios de la madre ${ }^{\star \star}$} \\
\hline Universitarios y/o superiores & $942(40,3)$ & $644(41,7)$ & $298(37,4)$ & \multirow{3}{*}{,016 } \\
\hline Secundarios/secundarios superiores & $331(21,4)$ & $156(19,6)$ & $487(20,8)$ & \\
\hline Básicos o sin estudios & $911(38,9)$ & $569(36,9)$ & $342(42,8)$ & \\
\hline \multicolumn{5}{|l|}{ Nivel de estudios del padre } \\
\hline Universitarios y/o superiores & $894(38,8)$ & $604(39,7)$ & $290(36,8)$ & \multirow{3}{*}{,258 } \\
\hline Secundarios/secundarios superiores & $466(20,2)$ & $310(20,4)$ & $156(19,8)$ & \\
\hline Básicos o sin estudios & $947(38,9)$ & $606(39,9)$ & $341(43,3)$ & \\
\hline \multicolumn{5}{|l|}{ Ingresos de la unidad familiar } \\
\hline Muy alto, alto, medio-alto & $780(33,3)$ & $510(32,8)$ & $270(33,8)$ & \multirow[t]{2}{*}{,677 } \\
\hline Medio, bajo, muy bajo & $1559(66,7)$ & $1034(66,5)$ & $525(65,6)$ & \\
\hline \multicolumn{5}{|l|}{ Frecuencia de salidas durante el último año } \\
\hline Varias veces/semana-una vez/mes & $1515(64,3)$ & $989(63,6)$ & $526(65,8)$ & \multirow{3}{*}{, 565} \\
\hline Una vez/mes/tres meses & $648(27,5)$ & $435(28,0)$ & $213(26,6)$ & \\
\hline Tres veces al año/nunca & $132(8,2)$ & $131(8,4)$ & $61(7,6)$ & \\
\hline \multicolumn{5}{|c|}{ Frecuencia del uso de alcohol durante el tiempo de ocio (DTO) } \\
\hline Siempre o muy a menudo & $1708(72,8)$ & $1127(72,6)$ & $581(73,1)$ & \multirow{3}{*}{,868 } \\
\hline A veces o pocas veces & $522(22,2)$ & $345(22,2)$ & $177(23,3)$ & \\
\hline Nunca & $117(5,0)$ & $80(5,2)$ & $37(4,7)$ & \\
\hline \multicolumn{5}{|c|}{ Importancia del uso de alcohol y otras drogas DTO } \\
\hline Indispensable, muy importante & $784(33,3)$ & $521(33,5)$ & $263(32,9)$ & \multirow{3}{*}{,924 } \\
\hline No muy importante & $780(33,1)$ & $516(33,2)$ & $264(33,0)$ & \\
\hline Indiferente & $789(33,5)$ & $517(33,3)$ & $272(34,0)$ & \\
\hline \multicolumn{5}{|l|}{ Frecuencia del uso de cannabis DTO*** } \\
\hline Siempre o muy a menudo & $173(7,5)$ & $99(6,5)$ & $74(9,5)$ & \multirow{3}{*}{$<, 001$} \\
\hline A veces o pocas veces & $725(31,5)$ & $451(29,7)$ & $174(35,2)$ & \\
\hline Nunca & $1400(60,9)$ & $969(63,8)$ & $431(55,3)$ & \\
\hline \multicolumn{5}{|c|}{ Frecuencia del uso de cocaína o anfetaminas DTO** } \\
\hline A veces o pocas veces & $137(5,8)$ & $73(4,8)$ & $64(8,3)$ & \multirow[t]{2}{*}{, 001} \\
\hline Nunca & $2145(94,0)$ & $1442(95,2)$ & $703(91,7)$ & \\
\hline \multicolumn{5}{|l|}{ Frecuencia del uso de benzodiazepinas DTO } \\
\hline Siempre o muy a menudo & $14(0,6)$ & $9(0,6)$ & $5(0,7)$ & \multirow{3}{*}{,952 } \\
\hline A veces o pocas veces & $38(1,7)$ & $26(1,7)$ & $12(1,6)$ & \\
\hline Nunca & $2223(97,7)$ & $1476(97,7)$ & $747(97,8)$ & \\
\hline
\end{tabular}

Nota. ILT: durante el tiempo libre ${ }^{\star} p<, 10 ;{ }^{* \star} p<, 05 ;{ }^{* \star *} p<, 001$. 
Tabla 2. Percepciones sobre violencia sexual e igualdad en el contexto de ocio nocturno.

\begin{tabular}{|c|c|c|c|c|c|}
\hline & Total & Mujeres & Hombres & & \\
\hline & N (\%) & N (\%) & N (\%) & OR bruta & OR ajustada ${ }^{a}$ \\
\hline \multicolumn{6}{|l|}{$\begin{array}{l}\text { Percepciones sobre igualdad, seguridad e impunidad en contextos de ocio } \\
\text { nocturno. «Los contextos/espacios de ocio nocturno... }\end{array}$} \\
\hline no son igualitarios para mujeres y hombres» ${ }^{\star \star \star}$ & $2024(86,0)$ & $1376(88,6)$ & $648(81,0)$ & $1,82(2,32-1,45)$ & $1,75(2,27-1,39)$ \\
\hline implican un menor nivel de seguridad» ${ }^{\star \star \star}$ & $1897(80,7)$ & $1305(84,1)$ & $592(74,1)$ & $1,85(1,5-2,28)$ & $1,80(1,45-2,23)$ \\
\hline 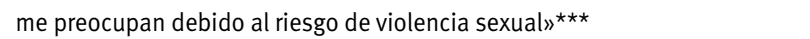 & $1446(61,5)$ & $1270(81,7)$ & $176(22,1)$ & $15,78(12,78-19,49)$ & $15,03(12,08-18,69)$ \\
\hline 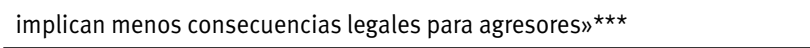 & $1417(61,4)$ & $979(64,7)$ & $438(55,1)$ & $1,49(1,25-1,78)$ & $1,62(1,35-1,94)$ \\
\hline \multicolumn{6}{|c|}{ Percepciones sobre las conductas que constituyen formas de violencia sexual. Esta conducta es violencia sexual: } \\
\hline Violación & $2322(98,6)$ & $1534(98,6)$ & $788(98,5)$ & $1,11(0,54-2,27)$ & $0,92(0,43-1,97)$ \\
\hline Tocamientos no consentidos & $2308(98,0)$ & $1529(98,3)$ & $779(97,4)$ & $1,58(0,89-2,84)$ & $1,61(0,86-3,01)$ \\
\hline Acorralamiento*** & $2143(91,0)$ & $1439(92,5)$ & $704(88,0)$ & $1,69(1,27-2,25)$ & $1,655(1,23-2,23)$ \\
\hline Comentarios sexuales invasivos ${ }^{\star \star \star}$ & $2134(90,6)$ & $1432(92,1)$ & $702(87,8)$ & $1,62(1,23-2,15)$ & $1,60(1,2-2,15)$ \\
\hline Insistencia frente a negativas $s^{\star \star \star}$ & $1909(81,1)$ & $1313(84,4)$ & $596(74,5)$ & $1,86(1,5-2,29)$ & $2,01(1,62-2,51)$ \\
\hline \multicolumn{6}{|l|}{ Normalización de la violencia sexual en los contextos de ocio nocturno CON } \\
\hline «las mujeres deben asumir los riesgos de sufrir violencia sexual en $\mathrm{CON} »{ }^{\star \star \star}$ & $223(9,5)$ & $122(7,9)$ & $101(12,7)$ & $0,59(0,44-0,78)$ & $0,53(0,4-0,71)$ \\
\hline $\begin{array}{l}\text { «las agresiones sexuales ocurren debido al mayor uso de drogas por } \\
\text { mujeres» }\end{array}$ & $454(19,4)$ & $278(17,9)$ & $176(22,1)$ & $0,77(0,63-0,96)$ & $0,70(0,56-0,88)$ \\
\hline 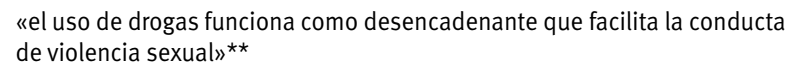 & $2140(91,2)$ & $1431(92,3)$ & $709(88,6)$ & $1,49(1,12-1,99)$ & $1,44(1,06-1,96)$ \\
\hline «prevenir el uso de drogas pondría fin a la violencia sexual en CON» & $170(7,2)$ & $108(6,9)$ & $62(7,8)$ & $0,89(0,64-1,23)$ & $0,85(0,6-1,19)$ \\
\hline \multicolumn{6}{|l|}{ Justificación de la violencia sexual. «Las relaciones sexuales están justificadas si... } \\
\hline una mujer se viste de manera provocativa» & $44(1,9)$ & $27(1,7)$ & $17(2,1)$ & $0,81(0,44-1,5)$ & $0,97(0,5-1,88)$ \\
\hline una mujer está bajo los efectos de las drogas»* & $62(2,6)$ & $34(2,2)$ & $27(3,5)$ & $0,62(0,37-1,02)$ & $0,58(0,34-0,99)$ \\
\hline $\begin{array}{l}\text { una mujer consiente abandonar el espacio de ocio nocturno con el } \\
\text { agresor }\end{array}$ & $151(6,5)$ & $83(5,4)$ & $68(8,6)$ & $0,61(0,44-0,85)$ & $0,57(0,4-0,8)$ \\
\hline
\end{tabular}

Nota. CON: contextos de ocio nocturno. ${ }^{\star} p<, 10 ;{ }^{\star \star} p<, 05 ;{ }^{\star \star \star} p<, 001$ a ajustado por edad, nivel de estudios de la madre, uso de cannabis y uso de cocaína/anfetaminas.

ocio nocturno como no igualitarios $(88,6 \%$ mujeres y $81 \%$ hombres, $p>, 001)$, el $80,7 \%$ los consideró menos seguros $(84,1 \%$ vs. $74,1 \%, p<, 001)$ y al $61,5 \%$ les preocupó el riesgo de violencia sexual en el contexto de ocio nocturno, una preocupación quince veces más frecuente entre las mujeres $(81,7 \%)$ que entre los hombres $(21,1 \%) \quad(p<$ ,001). En cuanto a la percepción sobre lo que constituye violencia sexual, el $98 \%$ de los encuestados consideró la violación y los tocamientos no consentidos como violencia sexual, sin diferencias significativas entre géneros. Se encontraron algunas diferencias respecto del acorralamiento, considerado como violencia sexual por el $91,0 \%$ de los encuestados $(92,5 \%$ de las mujeres y $88,0 \%$ de los hombres $(p<, 001))$, y los comentarios sexuales invasivos $(90,6 \%$; $92,1 \%$ de las mujeres y $87,8 \%$ hombres $(p<, 001))$. Cabe señalar la diferencia respecto de la percepción acerca de la insistencia frente a negativas $(81,1 \% ; 84,4 \%$ mujeres y $74,5 \%$ hombres, $(p<, 001))$. La consideración de este comportamiento como una forma de violencia es dos veces más frecuente entre las mujeres. Con respecto a la normalización de la violencia sexual en el contexto de ocio nocturno, un 9,5\% pensó que las mujeres tienen que asumir el riesgo de ser agredidas sexualmente $(12,7 \%$ hombres y $7,9 \%$ mujeres, $p<, 001)$ y el $19,4 \%$ vinculó la violencia sexual contra las mujeres en el contexto de ocio nocturno con el mayor consumo de drogas por parte de las mujeres, sin diferencias entre géneros. Aproximadamente el 91,2\% de los encuestados pensó que el consumo de drogas actúa como un desencadenante facilitador de las conductas sexuales violentas $(92,3 \%$ vs. $89,0 \% ; p<, 05)$, pero solamente el 7,2\% pensó que el cese del consumo de drogas acabaría con la violencia sexual en el contexto de ocio nocturno, sin diferencias significativas entre sexos. Asimismo, las relaciones sexuales forzadas contra mujeres en el contexto de ocio nocturno fueron justificadas por un $1,9 \%$ de los encuestados si una mujer viste de manera provocativa ( $\sin$ diferencias entre géneros), 2,6\% (3,5\% hombres y 2,2\% mujeres, $p<, 10)$, si una mujer está bajo los efectos de las drogas y el $6,5 \%(8,6 \%$ hombres y $5,4 \%$ mujeres, $p<, 05)$ si una mujer accede a irse con su agresor.

La Tabla 3 muestra las percepciones sobre el fenómeno de DFSA. Aproximadamente el 1,7\% de los encuestados estaría dispuesto a tener relaciones sexuales con una persona incapaz de expresar su consentimiento debido al consumo 
Tabla 3. Percepciones sobre DFSA.

\begin{tabular}{|c|c|c|c|c|c|}
\hline & Total & Mujeres & Hombres & & \\
\hline Disposición a realizar conductas DFSA & $\mathrm{N}(\%)$ & $\mathrm{N}(\%)$ & $\mathrm{N}(\%)$ & OR bruta & OR ajustada ${ }^{a}$ \\
\hline $\begin{array}{l}\text { «Estaría dispuesto a tener relaciones sexuales con alguien incapaz de expresar su } \\
\text { consentimiento debido al uso de drogas»» }\end{array}$ & $41(1,7)$ & $12(0,8)$ & $29(3,6)$ & $0,21(0,10-0,41)$ & $0,19(0,09-0,39)$ \\
\hline \multicolumn{6}{|l|}{ Prácticas de riesgo para victimización por DFSA en contextos de ocio nocturno CON } \\
\hline «Comparto mi propia bebida en $\mathrm{CON} »^{\star \star \star}$ & $1579(67,7)$ & $998(64,7)$ & $581(73,5)$ & $0,66(0,55-0,8)$ & $0,635(0,52-0,79)$ \\
\hline «Acepto bebidas con contenido desconocido en $\mathrm{CON}{ }^{\star \star \star}$ & $653(27,9)$ & $374(24,2)$ & $279(35,2)$ & $0,59(0,49-0,71)$ & $0,59(0,49-0,73)$ \\
\hline 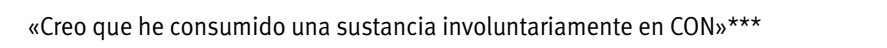 & $338(14,4)$ & $201(12,9)$ & $137(17,1)$ & $0,71(0,57-0,91)$ & $0,81(0,63-1,05)$ \\
\hline \multicolumn{6}{|c|}{ Tipo de consumo/sustancias implicadas en la mayoría de casos DFSA. «En la mayoría de casos DFSA... } \\
\hline ...la víctima consume drogas de manera voluntaria» ${ }^{\star \star \star}$ & $857(36,9)$ & $526(34,3)$ & $331(42,0)$ & $1,38(1,16-1,65)$ & $1,36(1,13-1,63)$ \\
\hline ...está involucrado el consumo de alcohol»*** & $787(33,4)$ & $479(31,3)$ & $308(39,2)$ & $0,71(0,59-0,85)$ & $0,76(0,63-0,91)$ \\
\hline ...está involucrado el consumo de escopolamina (Burundanga)»»** & $1119(48,4)$ & $768(50,3)$ & $351(44,7)$ & $0,71(0,59-0,85)$ & $0,76(0,63-0,91)$ \\
\hline \multicolumn{6}{|l|}{ Relación entre la víctima y el agresor en la mayoría de casos DFSA } \\
\hline "el agresor es alguien que la víctima conoce en la mayoría de casos DFSA» & $826(35,4)$ & $557(36,1)$ & $269(34,1)$ & $1,09(0,90-1,30)$ & $1,16(0,96-1,40)$ \\
\hline \multicolumn{6}{|l|}{ Percepción social del uso de drogas según género } \\
\hline 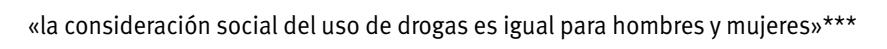 & $666(28,4)$ & $367(23,7)$ & $299(37,5)$ & $0,51(0,43-0,62)$ & $0,52(0,43-0,64)$ \\
\hline 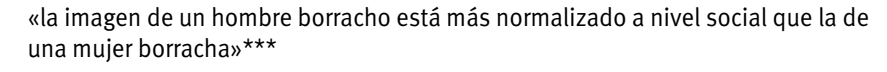 & $1912(81,3)$ & $1326(85,3)$ & $586(74,3)$ & $2,1(1,70-2,60)$ & $2,21(1,77-2,76)$ \\
\hline 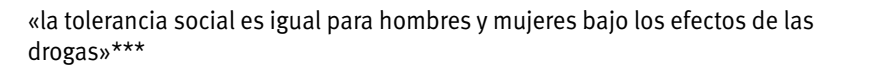 & $320(13,6)$ & $138(8,9)$ & $182(22,8)$ & $0,33(0,26-0,42)$ & $0,32(0,25-0,42)$ \\
\hline
\end{tabular}

Apoyo social en situaciones con posible riesgo de DFSA

«la sociedad penaliza a un hombre por intentar aprovecharse de una mujer que tiene dificultades motoras por el efecto de sustancias psicoactivas en $\mathrm{CON} »{ }^{\star \star \star}$

$1146(48,9) \quad 720(46,5) \quad 426(53,6) \quad 0,75(0,63-0,89) \quad 0,7(0,59-0,84)$

Nota. CON: en contextos de ocio nocturno. ${ }^{\star} p<, 10 ;{ }^{\star \star} p<, 05 ;{ }^{\star \star \star} p<, 001$ a ajustado por edad, nivel de estudios de la madre, uso de cannabis y uso de cocaína/anfetaminas.

de drogas. Esta disposición fue cinco veces mayor en los hombres $(3,6 \%)$ que en las mujeres $(0,8 \% ; p<, 001)$. Respecto de las prácticas de riesgo relacionadas con DFSA: el $67,7 \%$ comparte sus bebidas $(73,5 \%$ hombres y $64,7 \%$ mujeres, $p<, 001)$; el 27,7\% acepta bebidas con contenido desconocido $(35,2 \%$ vs. $24,2 \%, p<, 001)$ y el $14,4 \%$ cree que alguna vez ha ingerido ingerido una sustancia involuntariamente $(17,1 \%$ vs. $12,9 \%, p<, 001)$. En cuanto a conocimiento sobre sustancias DFSA, el 48,4\% pensó que la burundanga (escopolamina) estaba presente en la mayoría de los casos DFSA (44,7\% de hombres y $55,3 \%$ de mujeres, $p<, 001$ ) y el $34,0 \%$ pensó que el alcohol era la sustancia mayoritaria (39,2\% vs. $31,3 \%, p<, 001)$. Alrededor del 36,9\% de los encuestados pensó que DFSA suele ocurrir tras el uso voluntario de alguna sustancia por parte de la víctima $(42,0 \%$ hombres, $34,3 \%$ mujeres, $p<, 001$ ) y el $35,4 \%$ pensó que estas agresiones son cometidas por alguien que la víctima conoce personalmente (sin diferencias por género). En cuanto a la percepción social del consumo de drogas, el $37,5 \%$ de los hombres y el $23,7 \%$ de las mujeres $(\mathrm{p}<0,001)$ opinaron que la consideración social del consumo de drogas es igual para hombres y mujeres. Asimismo, el 22,8\% frente al 8,9\% ( $p<0,001)$ pensaba que hombres y mujeres bajo el efecto de las drogas enfrentan el mismo juicio social. Como tal, la percepción de que la tolerancia es la misma respecto de mujeres y hombres bajo el efecto de las drogas es tres veces más frecuente entre los hombres. Alrededor del 81,3\% pensó que la imagen de un hombre ebrio está más normalizada que la imagen de una mujer ebria, con la frecuencia siendo más de dos veces mayor entre mujeres $(85,3 \%)$ que entre hombres $(74,3 \%)$ $(p<, 001)$. Es más, el 28,7\% pensó que la opinión social sobre el consumo de drogas entre hombres es la misma que respecto del consumo de drogas entre mujeres $(37,5 \%$ hombres y $23,7 \%$ mujeres, $p<, 001)$. Por último, el 48,9\% cree que la sociedad penaliza a un hombre por intentar aprovecharse de una mujer que está experimentando dificultades motoras debido a los efectos provocados por las sustancias psicoactivas en el contexto de ocio nocturno; $53,6 \%$ hombres y $46,5 \%$ mujeres $(p<, 001)$.

\section{Discusión}

Una proporción preocupante de jóvenes todavía tiene unas creencias básicas incorrectas respecto de la violencia sexual, con marcadas diferencias entre géneros. Muchos internalizan el contexto de ocio nocturno como un espacio predominantemente masculino, donde las mujeres, simplemente por ser quienes son, deben asumir el riesgo de sufrir violencia sexual, así como un cierto grado de condena social si ocurre esta violencia. Además, los jóvenes tienes escasos conocimientos sobre DFSA o, lo que es peor, sus conceptos erróneos. 
Esta investigación se ha realizado en un contexto de jóvenes estudiantes universitarios, que consideramos representativos del grupo social joven, medio y medio-alto de la población española. En este sentido, las diferencias basadas en género fueron más notables a nivel sociodemográfico que a nivel de ocio nocturno. Sin embargo, a pesar de la representatividad del contexto, la muestra no es probabilística, una limitación derivada de las dificultades para investigar este tema. Las mujeres eran un poco más jóvenes que los hombres, sus padres tenían un nivel de estudios ligeramente superior y presentaban un patrón de ocio nocturno más cercano al patrón masculino que el de las generaciones anteriores. Estos datos muestran como se ha alcanzado una convergencia entre hombres y mujeres en cuanto a patrones de consumo (Calafat et al., 2009) en un corto período de tiempo (Cortés Tomás, Espejo Tort, Martín del Río y Gómez Iñíguez, 2010). Sin embargo, aunque un número cada vez mayor de mujeres ingresa a la vida nocturna, persisten graves desigualdades de género. (Calafat et al., 2009). Tal y como indicaban las hipótesis planteadas inicialmente, las percepciones de igualdad, seguridad e impunidad en los contextos de ocio nocturno difieren entre mujeres y hombres. Tan solo el $11,4 \%$ y el $19,0 \%$, respectivamente, piensan que los espacios de ocio nocturno son igualitarios, en coherencia con otros estudios españoles (European Union Agency for Fundamental Rights, 2014; Government Delegation against Gender Violence, 2018). Asimismo, nuestros resultados muestran una importante percepción femenina del ocio nocturno como un contexto caracterizado por un bajo nivel de seguridad personal. Más del $80 \%$ de las mujeres están preocupadas por el riesgo de violencia sexual en el contexto de ocio nocturno (quince veces más que los hombres). Esta percepción limita la libertad de acción y movimiento de las mujeres. De hecho, en un estudio relacionado, se encontró que hasta la mitad de las mujeres europeas evitaba algunas situaciones o lugares por temor a sufrir una agresión sexual (European Union Agency for Fundamental Rights, 2014). En consecuencia, las mujeres podrían estar sufriendo más consecuencias negativas en este proceso de convergencia que se está produciendo en contextos de ocio nocturno (Calafat et al., 2009).

Casi todos los participantes en nuestra muestra consideraron determinadas conductas, como la violación y los tocamientos no consentidos, como violencia sexual. Sin embargo, la opinión varió en mayor medida respecto de si otras conductas menos explícitas, como acorralamiento o comentarios sexuales invasivos, son formas de violencia sexual, tal y como han observado otros estudios anteriormente. (Government Delegation against Gender Violence, 2018; McMahon y Farmer, 2011). Asimismo, tal y como se planteó a través de una de las hipótesis iniciales, existe una brecha social entre géneros en lo que respecta a la identificación de determinados comportamientos como violencia sexual. Estas diferencias son más marcadas en cuanto a la consideración de la insistencia frente a negativas como una forma de violencia, que se observó como dos veces más frecuente entre mujeres. Esta percepción errónea tiene dos consecuencias. posibles víctimas, las percepciones sobre lo que constituye o no violencia condicionan su propia capacidad para reconocerse a sí mismas como tal. (Prego-Meleiro et al., 2020a). Muchas personas no identifican sus propias experiencias sexuales no consentidas como un delito, sino que las conceptualizan como un problema de comunicación, sexo malo o simplemente no saben cómo identificarlas (Bondurant, 2001; Fisher et al., 2003; Littleton, Axsom, Breitkopf y Berenson, 2006). Esta dificultad es más común entre las víctimas de agresión sexual que habían consumido alcohol antes de que ocurriese la agresión (Bondurant, 2001; Littleton, Axsom y Grills-Taquechel, 2009). Estas víctimas sienten que sus experiencias no representan una «violación real» (Littleton y Axsom, 2003). Asimismo, respecto a la cultura de protección de los agresores, la normalización de actitudes, creencias y experiencias de socialización distorsionadas resulta en conductas sexuales agresivas (Benson, Charlton y Goodhart, 1992; Margolin, Miller y Moran, 1989).

El consumo de drogas juega un papel importante en la violencia sexual desde dos perspectivas diferentes. Primero, las drogas, especialmente el alcohol, están muy vinculadas e integradas en la actividad recreativa del contexto de ocio nocturno (Calafat et al., 2009; Hughes et al., 2011; Olszewski, 2008; Romo-Avilés, García-Carpintero y Pavón-Benítez, 2019). De hecho, se observó que el consumo de alcohol es indispensable o muy importante para un tercio de los encuestados durante sus actividades de ocio nocturno, una proporción mayor la observada por estudios previos en el mismo contexto (Calafat et al., 1999). Segundo, el consumo de drogas parece ser una de las principales causas de violencia sexual para muchos jóvenes. El 20\%, aproximadamente, atribuye la violencia sexual contra las mujeres en el contexto de ocio nocturno al mayor uso de drogas en la actualidad por parte de ellas. Esta percepción implica culpar a las mujeres por la violencia que sufren y puede explicarse por la adhesión social a los estereotipos de género, que penalizan más el consumo femenino debido a la transgresión de los roles tradicionalmente asignados a las mujeres. Según esta creencia, beber alcohol antes de la agresión provoca que la víctima sea percibida como promiscua responsable de la agresión (Grubb y Turner, 2012). Estas observaciones son coherentes con una de nuestras hipótesis originales: las mujeres experimentan una falta de apoyo social en contextos de ocio nocturno, resultado de las diferentes percepciones sociales sobre el consumo de drogas en función del género. Por último, el uso de drogas se considera en gran medida como un factor desencadenante de la violencia sexual. En este punto, nos centramos solamente en el consumo masculino, dado que las conductas de violencia sexual principalmente se ejecutan por par- 
te de hombres contra mujeres (Organización Mundial de la Salud, 2002). Sin embargo, la violencia sexual en el contexto de ocio nocturno no debe considerarse únicamente en función del consumo de drogas. En este sentido, aunque un $90 \%$ de los encuestados piensa que el uso de drogas facilita la violencia sexual, solo el $7,2 \%$ cree que detener el consumo acabaría con la violencia. La violencia cultural contra las mujeres es un componente esencial de la violencia sexual, configurando así una realidad socioestructural que apoya y justifica la conducta violenta. En España, alrededor del $50 \%$ de los hombres y el $45 \%$ de las mujeres piensan que un hombre viola a una mujer debido al alcohol (Government Delegation against Gender Violence, 2018). Sin embargo, esta declaración puede interpretarse como si la población de alguna manera estuviera «eximiendo» a los agresores de sus acciones. De hecho, una persona puede compartir sus percepciones para justificar la violencia de una manera sutil y nunca culpar directamente a la víctima. Por ejemplo, cuando se piensa que las agresiones ocurren debido a la forma en que se viste una mujer o porque las víctimas consumen alcohol u otras drogas (Lorenz y Ullman, 2016), algo que este estudio fue el caso para un gran número de hombres. Asimismo, es sorprendente encontrar diferencias entre sexos en cuanto a la disposición para tener relaciones sexuales con una persona incapaz de expresar el consentimiento debido al consumo de drogas. En este sentido, las observaciones apoyan nuestra hipótesis anterior de que los hombres muestran una mayor disposición que las mujeres para mantener relaciones sexuales con alguien que no puede expresar su consentimiento debido a las drogas. Así, la disposición masculina alcanza el $3,6 \%$, una cifra casi cinco veces superior a la de las mujeres, aunque similar a las estimaciones de otros países (Victorian Health Promotion Foundation, 2015). En este sentido, estudios previos sugirieron que el consumo de alcohol por parte de las víctimas de una agresión sexual que implica alcohol con frecuencia es fomentado por otra persona, a menudo el agresor (Lynch, Wasarhaley, Golding y Simcic, 2013). Cabe señalar que, en situaciones de citas y encuentros sexuales casuales, los objetivos sexuales con más frecuencia son la motivación principal entre los hombres que entre las mujeres (Bradshaw, Kahn y Saville, 2010). De acuerdo con esta observación, proporciones significativas de hombres estudiantes universitarios admiten haber animado a sus contrapartes femeninas a consumir alcohol en un intento por lograr tener sexo con ellas (Lynch et al., 2013; Romero-Sánchez y Megías, 2010; Sipsma, Carrobles, Montorio y Everaerd, 2000). Del mismo modo, las mujeres universitarias informan sufrir agresiones sexuales después de que otra persona les emborrachara con el doble de frecuencia que después de haber sido forzadas. (Tyler, Hoyt y Whitbeck, 1998).

En este estudio, tres mitos se relacionaron con el fenómeno DFSA, lo que se ajusta a otra de nuestras hipótesis origina- les: existen varios mitos o conceptos erróneos generalizados sobre el fenómeno DFSA, que afectan de manera significativa la concepción social de esta forma de violencia sexual. Primero, la mayoría de los jóvenes cree que las agresiones ocurren después de la administración subrepticia de algún tipo de sustancia por parte del agresor a la víctima. Sin embargo, es mucho más frecuente que las víctimas consuman drogas voluntariamente antes de la agresión (García-Caballero, Quintela-Jorge y Cruz-Landeira, 2017; Hagemann, Helland, Spigset, Espnes, Ormstad y Schei, 2013; Scott-Ham y Burton, 2005). Además, el 48,4\% cree que la burundanga (escopolamina) es la sustancia involucrada en la mayoría de los casos DFSA, disminuyendo esta cifra al $34 \%$ para las personas que piensan que el alcohol está involucrado. Sin embargo, los casos DFSA confirmados que implican el uso de la escopolamina en España son anecdóticos (Gomila, Puiguriguer y Quesada, 2016), mientras que existe evidencia significativa de la involucración del alcohol en casos DFSA a nivel nacional (García-Caballero et al., 2017; Navarro y Vega, 2013; Xifró-Collsamata et al., 2015) e internacional (Hagemann et al., 2013; Scott-Ham y Burton, 2005). Ambos mitos están relacionados, ya que los medios de comunicación han contribuido a diseminar la idea de la administración encubierta de «drogas para la violación», tales como flunitrazepam, gamma-hidroxibutirato (GHB) (Hagemann et al., 2013) y escopolamina (Gomila et al., 2016). Llama la atención la mayor creencia de estos mitos en las mujeres y puede ser explicado por su mayor preocupación respecto de sufrir violencia sexual. Esto puede motivarlas a tener más en cuenta estas historias de miedo relativas a la administración subrepticia de determinadas sustancias (Prego-Meleiro et al., 2020a). Por último, otro error identificado es que la mayoría de los encuestados piensa que, en gran parte de los casos, los agresores no son conocidos por la víctima, lo cual no suele ser el caso (Panyella-Carbó, Agustina y Martin-Fumadó, 2019; Prego-Meleiro et al., 2020a).

Es muy importante tener en cuenta que los mitos y las percepciones erróneas obstaculizan o impiden a las personas que sufren ADSF de reconocerse a sí mismas como víctimas, algo crítico cuando se trata de informar de la agresión y buscar ayuda (Lorenz y Ullman, 2016; Prego-Meleiro et al., 2020a). Sin embargo, incluso si se identifican como tal, la decisión de denunciar un incidente está influenciada por las reacciones sociales negativas que las víctimas piensan se encontrarán (Burt, 1980; Heider, 1958; Lerner, 1980). Cuando una agresión sexual involucró el uso de drogas por parte de la víctima, las reacciones sociales al incidente dependen de las percepciones culturales sobre el uso de drogas, que varían según el género del consumidor. (Prego-Meleiro et al., 2020a). Las mujeres jóvenes tienden a creer que el consumo de drogas por parte de las mujeres está socialmente más penalizado que el consumo de drogas por parte de los hombres. La percepción de que existe igualdad de tolerancia para mujeres y hombres drogados es tres veces más fre- 
cuente entre los hombres. Del mismo modo, la creencia de que la imagen de un hombre ebrio está más normalizada que la de una mujer ebria es dos veces frecuente entre las mujeres que entre los hombres. Así, como sugerimos anteriormente, las mujeres experimentan una falta de apoyo social en los contextos de ocio nocturno, resultado de las diferentes percepciones sociales sobre el consumo de drogas en función del género. Las reacciones sociales negativas hacia las víctimas afectan negativamente su recuperación (Relyea y Ullman, 2015), lo que implica un mayor riesgo de revictimización (Lorenz y Ullman, 2016). La ausencia de un apoyo social adecuado también puede conducir a las víctimas a no reconocer la necesidad de tomar precauciones contra futuras agresiones (Littleton et al., 2009) o a refugiarse en el alcohol como una estrategia de afrontamiento del dolor emocional (Lorenz y Ullman, 2016). Esta situación puede encerrar a las víctimas en una espiral de revictimización cíclica (Prego-Meleiro et al., 2020a). En este sentido, cuando preguntamos sobre el apoyo social esperado en una situación de riesgo de DFSA solo el 48,9\% cree que la sociedad penalizaría a un hombre por intentar aprovecharse de una mujer que está experimentando dificultades motoras debido a los efectos provocados por las sustancias psicoactivas en el contexto de ocio nocturno. Esta percepción de falta de apoyo social está más extendida entre las mujeres y se asocia a la mayor percepción femenina de impunidad para los agresores en contextos de ocio nocturno. Finalmente, deben tenerse en cuenta los ajustes estadísticos aplicados. El equilibrio entre mujeres y hombres no se alteró cuando los análisis se realizaron ajustando los datos por variables proxy para el nivel de estudios y la frecuencia de salidas. Por lo tanto, las diferencias observadas no son atribuibles a otros factores distintos al género.

\section{Limitaciones}

Este estudio tiene varias limitaciones. Aunque se utilizó un método de muestreo no probabilístico, la representatividad de la muestra es válida, dado que la composición es coherente con el perfil sociodemográfico de cualquier otra universidad española. Asimismo, los resultados provienen de un grupo poblacional con un nivel social medio a alto, por lo que el error podría ser considerablemente mayor en otros grupos poblacionales. Además, este estudio no utilizó preguntas validadas debido a la naturaleza pionera de este trabajo en España, donde a pesar de la relevancia de este fenómeno, las investigaciones sobre violencia sexual y DFSA han sido limitadas. En consecuencia, los parámetros estudiados aún no se han incluido en escalas psicométricas.

\section{Conclusiones}

Un segmento significativo de estudiantes universitarios no percibe determinadas formas de violencia sexual y comparte varias actitudes y percepciones que justifican la violencia sexual. El uso de drogas, especialmente alcohol, es ampliamente considerado como un factor que origina y justifica la violencia sexual contra las mujeres, siendo esta idea particularmente prevalente entre los hombres. Esta tolerancia hacia la violencia sexual lleva a las mujeres a sufrir situaciones de desventaja, en particular en contextos de ocio nocturno, donde el consumo de drogas está ampliamente extendido. En este sentido, los hallazgos de este estudio son de utilidad para diseñar esfuerzos de prevención dirigidos a aumentar la concienciación social de adolescentes y jóvenes contra la violencia sexual en contextos de ocio nocturno. Estos esfuerzos deben concentrarse en desmitificar el fenómeno DFSA, potenciar el apoyo social a las víctimas antes, durante y después de la agresión y evitar culpabilizarlas. La identificación de conceptos erróneos sobre la violencia sexual debe propiciar el desarrollo de programas de intervención preventivos e informativos, con un especial enfoque en los hombres, con el objetivo de promover la generación de espacios equitativos y seguros. Todas las formas de violencia sexual deben estar bien definidas y entendidas por los posibles agresores, las víctimas y la sociedad en su conjunto. Es necesario un cambio de mentalidad para conseguir una sociedad más solidaria y activa contra la violencia sexual, concretamente frente al fenómeno DFSA.

\section{Reconocimientos}

Los autores desean expresar su gratitud al Plan Nacional sobre Drogas del Ministerio de Sanidad, Servicios Sociales e Igualdad por su apoyo económico al proyecto MSCBS-PNSD-2018/032. Asimismo, los autores agradecen a la Universidad de Alcalá el apoyo a los proyectos UAH/ EV1024 y UAH/EV951. Además, se agradece al Instituto Universitario de Investigación en Ciencias Policiales por el proyecto IUICP/PI2019/006. Por último, PPM agradece a la Universidad de Alcalá una beca predoctoral.

\section{Conflicto de intereses}

Los autores declaran la inexistencia de conflicto de interés.

\section{Referencias}

Advisory Council on the Misuse of Drugs. (2007). Drug Facilitated Sexual Assault. London, United Kingdom: Author. 18 p. Recuperado de https://assets.publishing. service.gov.uk/government/uploads/system/uploads/ attachment_data/file/119111/ACMDDFSA.pdf.

Anderson, L. J., Flynn, A. y Pilgrim, J. L. (2017). A global epidemiological perspective on the toxicology of drug-facilitated sexual assault: A systematic review. Journal of Forensic and Legal Medicine, 47, 46-54. doi:10.1016/j. jflm.2017.02.005. 
Benson, D., Charlton, C. y Goodhart, F. (1992). Acquaintance rape on campus: A literature review. Journal of American College Health, 40, 157-165. doi:10.1080/07448 481.1992.9936277.

Bird, E. R., Gilmore, A. K., George, W. y Lewis, M. A. (2015). The role of social drinking factors in the relationship between incapacitated sexual assault and drinking before sexual activity. Addictive Behaviors, 52, 28-33. doi:10.1016/j.addbeh.2015.08.001.

Bondurant, B. (2001). University women's acknowledgment of rape: Individual, situational, and social factors. Violence Against Women, 7, 294-314. doi:10.1177/107780 1201007003004.

Bradshaw, C., Kahn, A. y Saville, B. (2010). To hook up or date: Which gender benefits? Sex Roles, 62, 661-669. doi:10.1007/s11199-010-9765-7.

Burt, M. R. (1980). Cultural myths and supports for rape. Journal of Personality and Social Psychology, 38, 217-230. doi:10.1037//0022-3514.38.2.217.

Calafat, A., Bohrn, K., Juan, M., Kokkevi, A., Maalsté, N., Mendes, F.,... Zavatti, P. (1999). Nightlife in Europe and Recreative Drug Use. Valencia, Spain: IREFREA. 242p. Recuperado de http://www.irefrea.eu/uploads/PDF/ Calafat\%20et\%20al_1999_SONAR\%2098.pdf.

Calafat, A., Fernandez, C. F., Juan M., Bellis M., Bohrn K., Hakkarainen P.,... Zavatti, P. (2003). Enjoying the nightlife in Europe: The role of moderation. Palma de Mallorca, Spain: IREFREA. 373 p. Recuperado de https://pdfs.semanticscholar.org/3578/e10b306fbabf1741d9b4dec6dd1ad3d917a9.pdf?_ga=2.22419698.859655376.1572901750334181427.1569319826.

Calafat, A., Juan, M., Becona, E., Mantecon, A. y Ramon, A. (2009). Sexualidad de riesgo y consumo de drogas en el contexto recreativo. Una perspectiva de género. Psicothema, 21, 227-233.

Carey, K. B., Durney, S. E., Shepardson, R. L. y Carey, M. P. (2015). Incapacitated and forcible rape of college women: Prevalence across the first year. Journal of Adolescent Health, 56, 678-680. doi:10.1016/j.jadohealth.2015.02.018.

Cortés Tomás, M., Espejo Tort, B. E., Martín del Río, B. y Gómez Iñíguez, C. (2010). Different typologies of alcohol consumers in the practice of the "botellon" in three Spanish cities. Psicothema, 22, 363. Recuperado de https://www.ncbi.nlm.nih.gov/pubmed/20667261.

European Union Agency for Fundamental Rights. (2014). Violence against women: An EU-wide survey. Results at a glance. Vienna, Austria: Author. 44p. Recuperado de https://fra.europa.eu/sites/default/files/fra-2014-vawsurvey-at-a-glance-oct14_en.pdf.

Fisher, B. S., Daigle, L. E., Cullen, F. T. y Turner, M. G. (2003). Reporting sexual victimization to the police and others: Results from a national-level study of co- llege women. Criminal Justice and Behavior, 30, 6-38. doi:10.1177/0093854802239161.

Folgar, M. I., Rivera, F. F., Sierra, J. C. y Vallejo-Medina, P. (2015). Binge drinking: Conductas sexuales de riesgo y drogas facilitadoras del asalto sexual en jóvenes españoles. Suma Psicológica, 22, 1-8. doi:10.1016/j.sumpsi.2015.05.001.

Galtung, J. (1990). Cultural violence. Journal of Peace Research, 27, 291-305. Recuperado de https://www.galtung-institut.de/wp-content/uploads/2015/12/Cultural-Violence-Galtung.pdf.

García-Caballero, C., Quintela-Jorge, O. y Cruz-Landeira, A. (2017). Alleged drug-facilitated sexual assault in a Spanish population sample. Forensic Chemistry, 4, 61-66. doi:10.1016/j.forc.2017.02.009.

Gilbert, J. y Pearson, E. (2003). Cultura y políticas de la música dance: Disco, hip-hop, house, techno, drum'n'bass and garage. Barcelona: Paidós.

Gomila, M., Puiguriguer, F. y Quesada, R. (2016). Drug facilitated crime using burundanga: First analytical confirmation in Spain. Medicina Clínica, 147, 421. doi:10.1016/j.medcli.2016.06.025.

Government Delegation against Gender Violence. (2015). Macroencuesta de violencia contra la mujer 2015. Madrid, Spain: Ministry of Health, Social Services and Equality. 468 p. Recuperado de http://www.violenciagenero. igualdad.mpr.gob.es/violenciaEnCifras/estudios/colecciones/pdf/Libro_22_Macroencuesta2015.pdf.

Government Delegation against Gender Violence. (2018). Percepción social de la violencia sexual. Madrid, Spain: Ministry of Health, Social Services and Equality. 135 p. Recuperado de http://www.violenciagenero.igualdad. mpr.gob.es/violenciaEnCifras/estudios/colecciones/ estudio/Libro25_Violencia_Sexual.htm.

Government Delegation for National Plan on Drugs. (2018). Encuesta sobre alcohol y otras drogas en españa (EDADES) (1995-2017). Madrid, Spain: Ministry of Health, Social Services and Equality. 137 p. Recuperado de http:/ / www.pnsd.mscbs.gob.es/profesionales/sistemasInformacion/sistemaInformacion/pdf/EDADES_2017_Informe.pdf.

Grubb, A. y Turner, E. (2012). Attribution of blame in rape cases: A review of the impact of rape myth acceptance, gender role conformity and substance use on victim blaming. Aggression and Violent Behavior, 17, 443-452. doi:10.1016/j.avb.2012.06.002.

Hagemann, C. T., Helland, A., Spigset, O., Espnes, K. A., Ormstad, K. y Schei, B. (2013). Ethanol and drug findings in women consulting a sexual assault center - associations with clinical characteristics and suspicions of drug-facilitated sexual assault. Journal of Forensic and Legal Medicine, 20, 777-784. doi:10.1016/j.jflm.2013.05.005.

Heider, F. (1958). The psychology of interpersonal relations. New York: Wiley. 
Hellmann, D. E., Kinninger, M. W. y Kliem, S. (2018). Sexual violence against women in Germany: Prevalence and risk markers. International Journal of Environmental Research and Public Health 15:1613-1631. doi:10.3390/ ijerph15081613.

Hughes, K., Quigg, Z., Eckley, L., Bellis, M., Jones, L., Calafat, A.,... van Hasselt, N. (2011). Environmental factors in drinking venues and alcohol-related harm: The evidence base for European intervention. Addiction, 106, 37-46. doi:10.1111/j.1360-0443.2010.03316.x.

Koss, M. P., Abbey, A., Campbell, R., Cook, S., Norris, J., Testa, M.,... White, J. (2006). The sexual experiences long form victimization (SES-LFV). Arizona: Tucson, AZ: University of Arizona. Recuperado de http://www.midss. org/content/sexual-experiences-survey-long-form-victimization-ses-lfv.

Krebs, C. P., Lindquist, C. H., Warner, T. D., Fisher, B. S. y Martin, S. L. (2007). The campus sexual assault (CSA) study. Washington D.C., United States of America: National Institute of Justice. 111p. Recuperado de https:/ / www.ncjrs.gov/pdffiles1/nij/grants/221153.pdf.

Lawyer, S., Resnick, H., Bakanic, V., Burkett, T. y Kilpatrick, D. (2010). Forcible, drug-facilitated, and incapacitated rape and sexual assault among undergraduate women. Journal of American College Health, 58, 453-460. doi:10.1080/07448480903540515.

Lerner, M. J. (1980). Belief in a just world: A fundamental delusion. Boston: Springer.

Littleton, H. y Axsom, D. (2003). Rape and seduction scripts of university students: Implications for rape attributions and unacknowledged rape. Sex Roles, 49, 465475. doi:10.1023/A:1025824505185.

Littleton, H. L., Axsom, D., Breitkopf, C. R. y Berenson, A. (2006). Rape acknowledgment and post-assault experiences: How acknowledgment status relates to disclosure, coping, worldview, and reactions received from others. Violence and Victims, 21, 761-778. doi:10.1891/ vv-v21i6a006.

Littleton, H., Axsom, D. y Grills-Taquechel, A. (2009). Sexual assault victims' acknowledgment status and revictimization risk. Psychology of Women Quarterly, 33, 34-42. doi:10.1111/j.1471-6402.2008.01472.x.

Lorenz, K. y Ullman, S. (2016). Alcohol and sexual assault victimization: Research findings and future directions. Aggression and Violent Behavior, 31, 82-94. doi:10.1016/j. avb.2016.08.001.

Lynch, K. R., Wasarhaley, N. E., Golding, J. M. y Simcic, T. (2013). Who bought the drinks? Juror perceptions of intoxication in a rape trial. Journal of Interpersonal Violence, 28, 3205-3222. doi:10.1177/0886260513496900.

Margolin, L., Miller, M. y Moran, P. B. (1989). When a kiss is not just a kiss: Relating violations of consent in kissing to rape myth acceptance. Sex Roles, 20, 231-243. doi:10.1007/BF00287721.
McMahon, S. (2010). Rape myth beliefs and bystander attitudes among incoming college students. Journal of American College Health, 59, 3-11. doi:10.1080/07448481.201 0.483715 .

McMahon, S. y Farmer, G. L. (2011). An updated measure for assessing subtle rape myths. Social Work Research, 35, 71-81. doi:10.1093/swr/35.2.71.

Megías, J. L., Romero-Sánchez, M., Durán, M., Moya, M. y Bohner, G. (2011). Spanish validation of the acceptance of modern myths about sexual aggression scale (AMMSA). The Spanish Journal of Psychology, 14, 912-925. doi:10.5209/rev_SJOP.2011.v14.n2.37.

Mustaine, E. E. y Tewksbury, R. (2002). Sexual assault of college women: A feminist interpretation of a routine activities analysis. Criminal Justice Review, 27, 89-123. doi:10.1177/073401680202700106.

Navarro, E. E. y Vega, C. V. (2013). Drug facilitated sexual assault, detected at the institute of legal medicine of Alicante in the years 2009-2012. Gaceta Internacional de Ciencias Forenses, 8-15.

O’Neil, M. y Morgan, P. (2010). American perceptions of sexual violence: A FrameWorks research report. Washington, D.C., United States of America: FrameWorks Institute. 33 p. Recuperado de https://www.frameworksinstitute. org/assets/files/PDF_sexualviolence/AmericanPerceptionsofSexualViolence.pdf.

Observatorio Noctambul@s. (2017). $3^{\circ}$ informe anual 2015/2016. Barcelona, Spain: Fundación Salud y Comunidad. 118 p. Recuperado de https://www.drogasgenero.info/wp-content/uploads/3er-Informe-Anual-Observatorio-Noctambul@s-2015-2016.pdf.

Observatorio Noctambul@s. (2018). $4^{\circ}$ informe anual 2016/2017. Barcelona, Spain: Fundación Salud y Comunidad. 106 p. Recuperado de https://www. drogasgenero.info/wp-content/uploads/4InformeNoct_2016-201717.pdf.

Olszewski, D. (2008). Sexual assaults facilitated by drugs or alcohol. Lisbon, Portugal: European Monitoring Centre for Drugs and Drug Adiction. 19 p. Recuperado de http://www.emcdda.europa.eu/attachements.cfm/ att_50544_EN_TDS_sexual_assault.pdf.

Panyella-Carbó, M. N., Agustina, J. R. y Martin-Fumadó, C. (2019). Proactive versus opportunistic drug-facilitated sexual assault: Criminological analysis of sexual crimes facilitated by the use of psychoactive substances from a sample of court decisions. Revista Española De Investigación Criminológica: REIC, 17, 5. Recuperado de https:// dialnet.unirioja.es/servlet/articulo?codigo $=6877874$.

Prego-Meleiro, P., Montalvo, G., Quintela-Jorge, O. y García-Ruiz, C. (2020a). Increasing awareness of the severity of female victimization by opportunistic drug-facilitated sexual assault: A new viewpoint. Forensic Science International, 315, 110460. doi:10.1016/j.forsciint.2020.110460. 
Prego-Meleiro, P., Montalvo G., Quintela-Jorge O. y García-Ruiz C. (2020b). An ecological working framework as a new model for understanding and preventing the victimization of women by drug-facilitated sexual assault. Forensic Science International, 315, 110460. doi:10.1016/j. forsciint.2020.110438.

Relyea, M. y Ullman, S. (2015). Unsupported or turned against: Understanding how two types of negative social reactions to sexual assault relate to post-assault outcomes. Psychology of Women Quarterly, 39, 37-52. doi:10.1177/0361684313512610.

Resnick, H. S., Walsh, K., Schumacher, J. A., Kilpatrick, D. G. y Acierno, R. (2012). Prior substance abuse and related treatment history reported by recent victims of sexual assault. Addictive Behaviors, 38, 2074-2079. doi:10.1016/j.addbeh.2012.12.010.

Romero-Sánchez, M. y Megías, J. L. (2010). Alcohol use as a strategy for obtaining non-consensual sexual relations: Incidence in Spanish university students and relation to rape myths acceptance. The Spanish Journal of Psychology, 13, 864-874. doi:10.1017/S1138741600002511.

Romo-Avilés, N., García-Carpintero, M. A. y Pavón-Benítez, L. (2019). Not without my mobile phone: Alcohol binge drinking, gender violence and technology in the Spanish culture of intoxication. Drugs: Education, Prevention and Policy, 27, 154-164. doi:10.1080/09687637.2019.158 5759.

Sasson, S. y Paul, L. A. (2014). Labeling acts of sexual violence: What roles do assault characteristics, attitudes, and life experiences play? Behavior and Social Issues, 23, 35-49. doi:10.5210/bsi.v.23i0.5215.

Scott-Ham, M. y Burton, F. C. (2005). Toxicological findings in cases of alleged drug-facilitated sexual assault in the United Kingdom over a 3-year period. Journal of Clinical Forensic Medicine, 12, 175-186. doi:10.1016/j. jcfm.2005.03.009.

Sipsma, E., Carrobles I., Montorio, I. y Everaerd, W. (2000). Sexual aggression against women by men acquaintances: Attitudes and experiences among Spanish university students. The Spanish Journal of Psychology, 3, 14-27. doi:10.1017/S1138741600005503.

Tyler, K. A., Hoyt, D. R. y Whitbeck, L. B. (1998). Coercive sexual strategies. Violence and Victims, 13, 47.
Ullman, S. E. y Filipas, H. (2001). Predictors of PTSD symptom severity and social reactions in sexual assault victims. Journal of Traumatic Stress, 14, 369-389. doi:10.1023/A:1011125220522.

Ullman, S. E. y Najdowski, C. J. (2010). Understanding alcohol-related sexual assaults: Characteristics and consequences. Violence and Victims, 25, 29-44. doi:10.1891/0886-6708.25.1.29.

Valls, R., Puigvert, L., Melgar, P. y Garcia-Yeste, C. (2016). Breaking the silence at Spanish universities. Violence Against Women, 22, 1519-1539. doi:10.1177/1077801215627511.

Victorian Health Promotion Foundation. (2015). Young Australians' attitudes to violence against women: Findings from the 2013 National Community Attitudes towards Violence Against Women Survey for respondents 16-24 years. Melbourne, Australia: Author. Recuperado de https://tasa.org. $\mathrm{au} /$ wp-content/uploads/2015/11/SurveyReport_YoungPeople-attitudes-violence-against-women-1.pdf.

Organización Mundial de la Salud. (2002). World report on violence and health: Summary. Geneva, Switzerland: Author. 54 p. Recuperado de https://www.who.int/violence_injury_prevention/violence/world_report/en/ summary_en.pdf.

Organización Mundial de la Salud. (2013). Global and regional estimates of violence against women: Prevalence and health effects of intimate partner violence and non-partner sexual violence. Geneva, Switzerland: Author. 50 p. Recuperado de https://apps.who.int/iris/handle/10665/85239?locale-attribute $=$ es\&.

Organización Mundial de la Salud, United nations Office on Drugs and Crime y United Nations Development Programme. (2014). Global status report on violence prevention 2014. Geneva, Switzerland: Author. 292 p. Recuperado de https://www.who.int/violence_injury_prevention/ violence/status_report/2014/en/.

Xifró-Collsamata, A., Pujol-Robinat, A., Barbería-Marcalain, E., Arroyo-Fernández, A., Bertomeu-Ruiz, A., Montero-Núñez, F. y Medallo-Muñiz, J. (2015) . A prospective study of drug-facilitated sexual assault in Barcelona. Medicina Clínica, 144, 403-409. doi:10.1016/j.medcli.2014.11.026. 
